\title{
Formation and evolution of gas-rich dwarf galaxies
}

Y. Ascasibar ${ }^{1}$, M. Gavilán ${ }^{1}$, M. Mollá ${ }^{2}$ A. I. Díaz ${ }^{1}$

${ }^{1}$ Universidad Autónoma de Madrid (Spain) ${ }^{2}$ Centro de Investigaciones Energéticas y Medioambientales (Spain)

We propose a scenario for the formation and evolution of isolated gas-rich dwarf galaxies based on the formation of stars fueled by continuous accretion of primordial gas from the surrounding intergalactic medium.

A grid of evolutionary models has been computed, including chemical evolution and stellar population synthesis in a selfconsistent way. Each model is characterized by the total mass of primordial gas available to the galaxy, the collapse time, and the star formation efficiency. Solid tracks in the figures show the evolution of the models, and dots mark their final state.

Model predictions (elemental abundances, stellar and gas masses, photometric colors) are compared with a set of observations of dwarf irregular and blue compact dwarf galaxies obtained from the literature. dlrr, field and cluster BCDs are plotted as stars, open and closed squares, respectively.

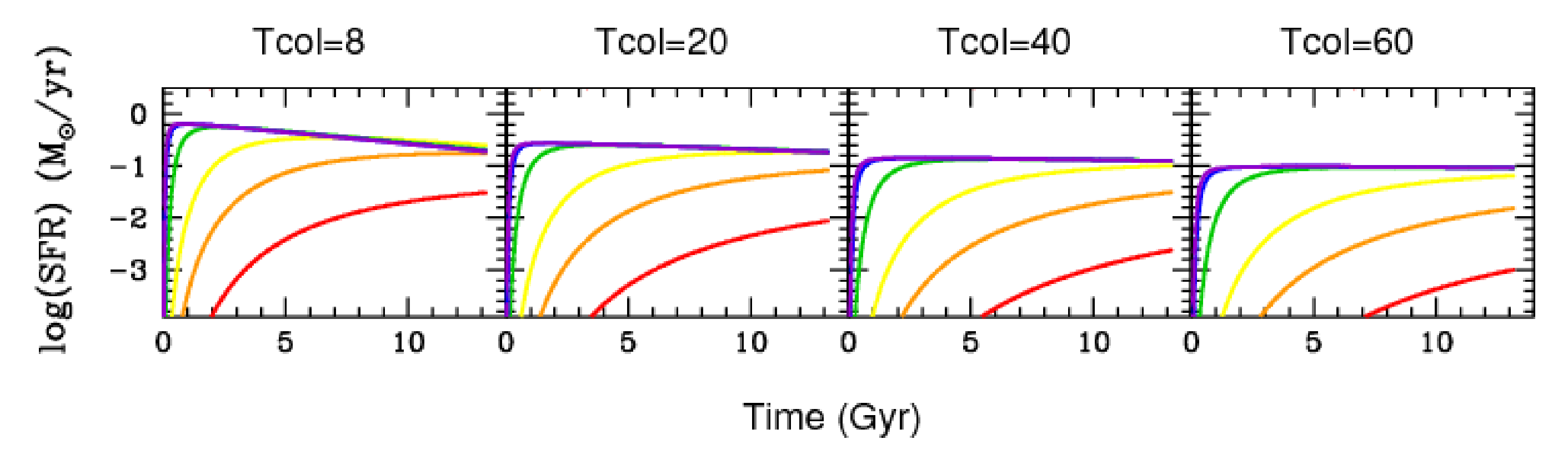

Evolution of the star formation rate for models with different collapse times and efficiencies.

High-efficiency models convert most of their gas into stars at early times. Low efficiencies yield higher gas fractions and star formation rates that peak at the present day.

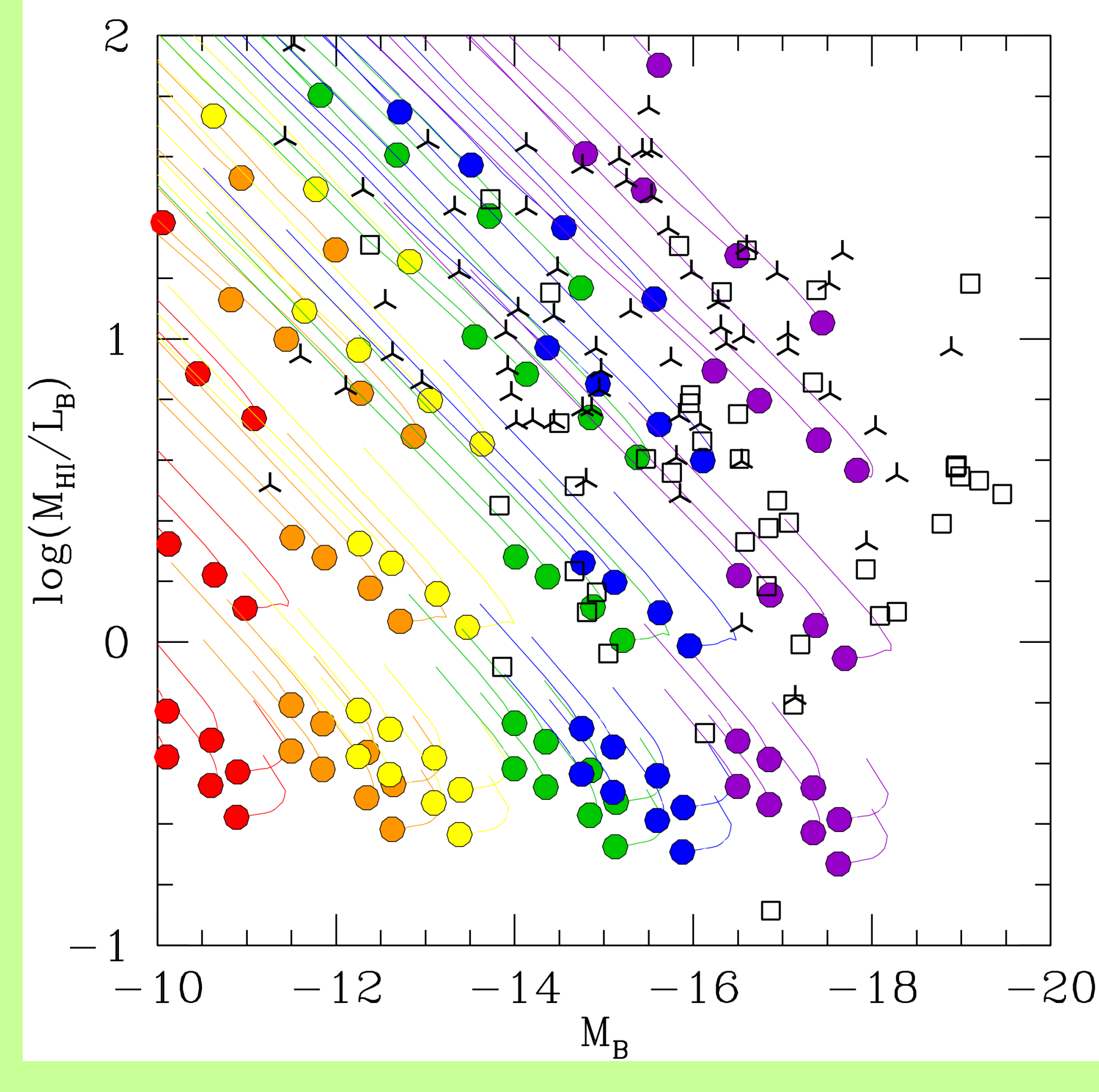

Mass-luminosity relation. The absolute $B$ magnitude of a galaxy roughly measures its stellar mass, while the ratio between total gas mass and stellar luminosity probes the star formation efficiency.

Higher efficiency models have lower gas content and provide a good fit to BCD galaxies, while lowefficiency models fit the less massive dlrr galaxies.

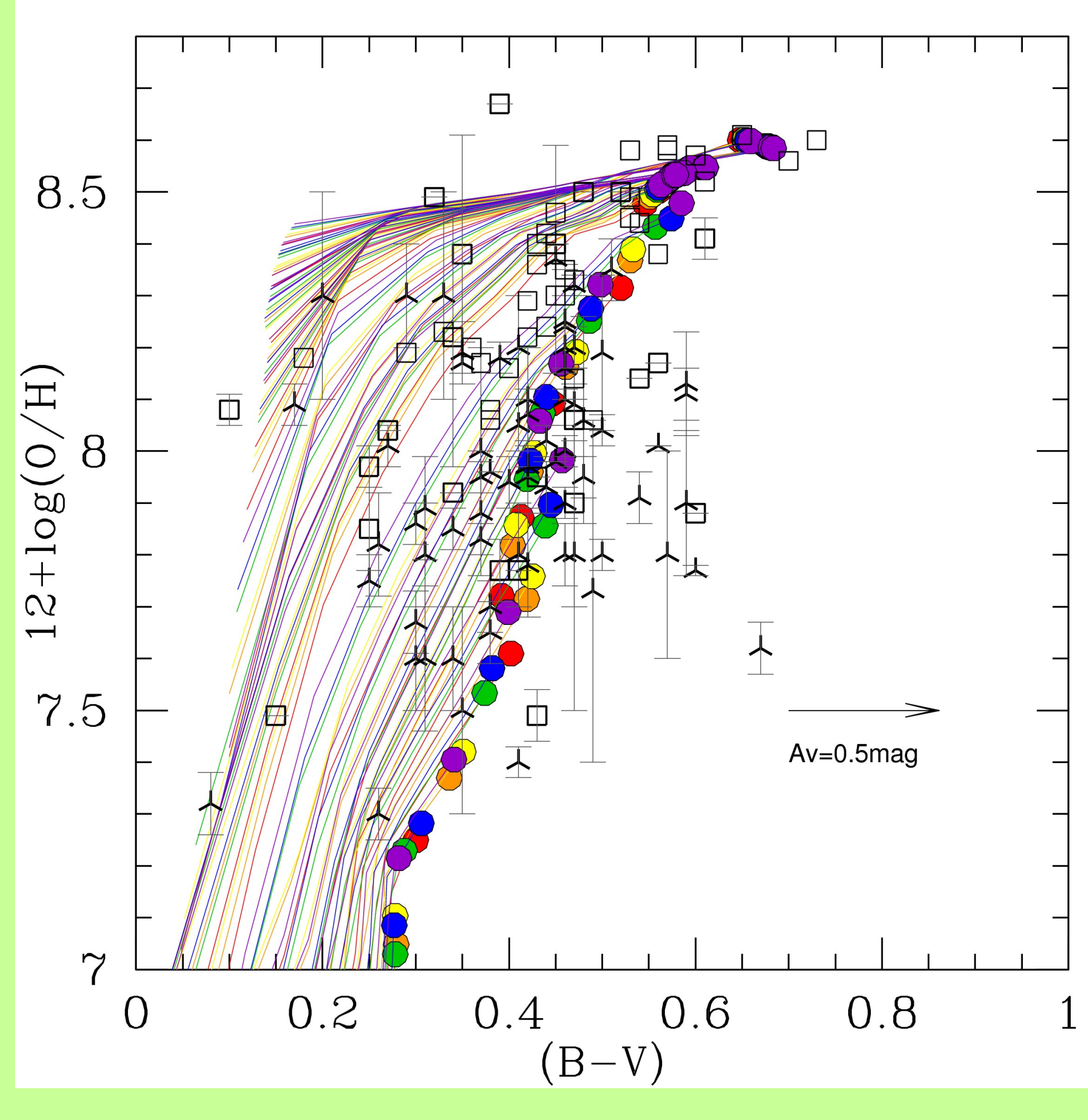

Oxygen appears early in the ISM, and thus its value grows abruptly in the beginning of the evolution, before the galaxy changes its color.

Nitrogen can be of primary or secondary origin, and it appears in the ISM with a delay of some dozens of millions of years.

Galaxies with low star formation efficiency are bluer, less chemically evolved, and show lower relative abundance of nitrogen.
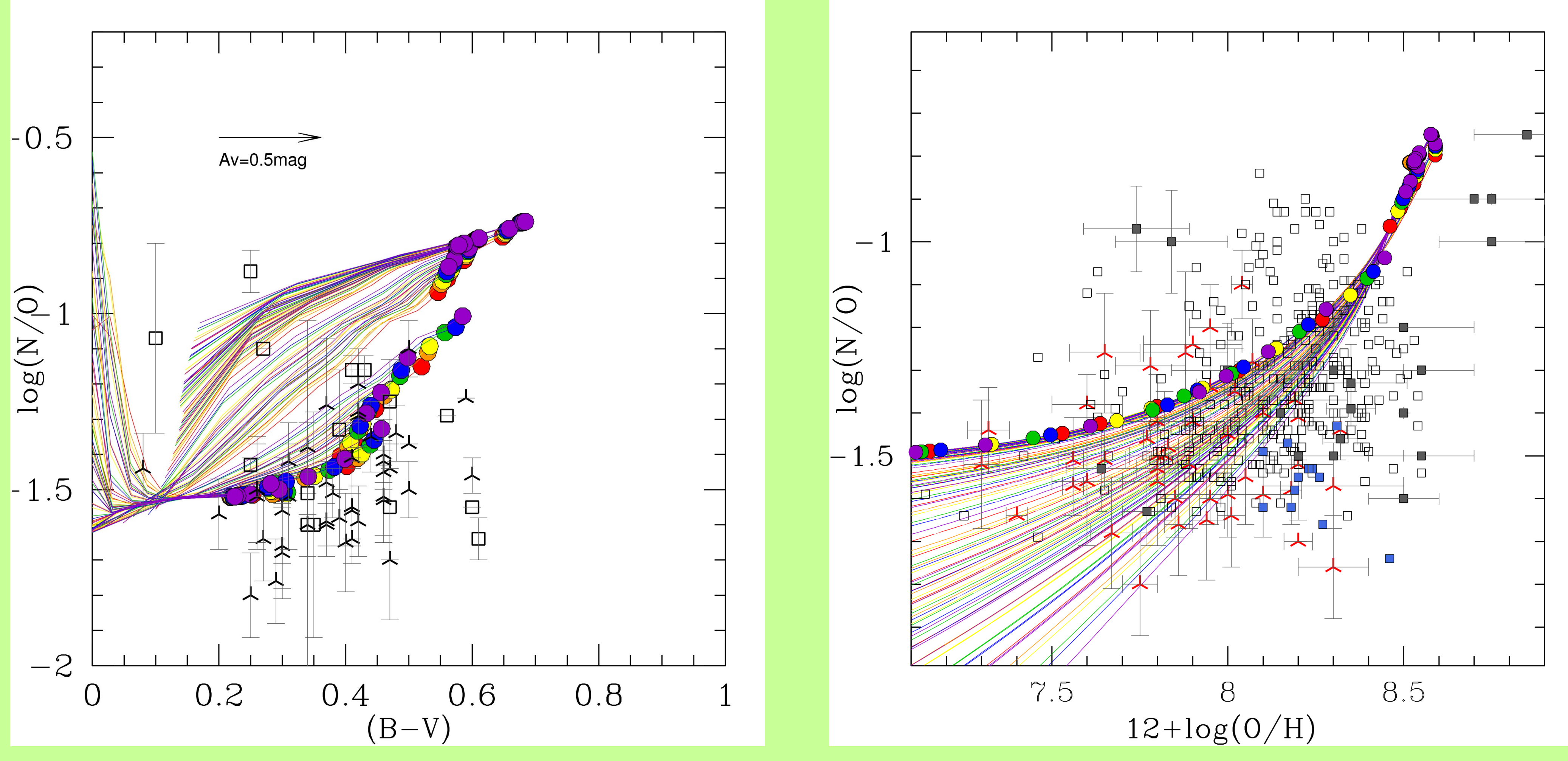

Combining the relations between gas mass, nitrogen and oxygen abundances, B-band luminosity and (B-V) color, we conclude that smaller galaxies must form less stars per unit gas mass than bigger systems.

Objects that show higher (N/O) than predicted by the models are good candidates for selective gas loss due to enriched galactic winds, whereas a low (N/O) ratio can be interpreted as evidence for a recent burst. 